\title{
Spatial inversion and P-parity nonconservation
}

\section{Chelnokov M.}

Department of Physics, Bauman Moscow State Technical University, Moscow, Russia;

E-mail: Chelnokov<l-chelnok@yandex.ru>;

The article deals with the mirror (looking glass) asymmetry of a three-dimensional Euclidean space which occurs where a real experimental situation is described by a set of real vectors and pseudovectors. The results are applied to the analysis of spatial parity non-conservation in processes of the particles.

In the article the well-known experiment of C.Wu (1957) on the detection of $P$-parity non-conservation in weak interaction processes is analyzed from a new point of view.

Keywords: symmetry mirror (looking glass), real vectors, pseudo-vectors, weak interaction, spatial inversion, spatial parity.

DOI: $10.18698 / 2309-7604-2015-1-112-120$

Mirror symmetry refers to discrete symmetries and, as it is known, it is the basis of the law of conservation of $P$-parity - spatial parity. The point of view was considered and remained obvious until 1956. In this year T.Lee and C.Yang in their famous article [1] carried out a systematic analysis of the law of conservation of $P$-parity in the processes with elementary particles and stated nonconservation of parity in the process caused by the weak interaction.

In 1957 group of physicists (Ambler, Hayward, Hopps and Hudson) under the leadership of C.Wu carried out a brilliant experiment [2] which showed $P$-parity is not conserved in the $\beta$ - decay of cobalt ${ }_{27} \mathrm{Co}^{60}$ caused by the weak interaction.

For the explanation of this phenomenon two hypotheses were suggested: the hypothesis of mirror asymmetry of the three-dimensional Euclidean space, and the hypothesis of the combined $C P$-parity in which the particles of the ordinary world are replaced by antiparticles behind the mirror. The second hypothesis was proposed by Wigner, Lee, Yang and Landau [3, 4].

The first hypothesis was not developed and it was almost forgotten. But the second hypothesis received the right for existence. But, firstly, it is a just a hypothesis that can not be tested directly, as it is hardly possible to imagine, how to detect antiparticles behind the mirror. This hypothesis seems to be initially confirmed, but only as it saves mirror symmetry.

Secondly, even the salvation proved to be illusory and of short duration, as 7 years later, in 1964, the experiment of Christensen and his colleagues [5] discovered nonconservation of $C P$ parity in the decays of kaons. The problem has been discussed and is being discussed in many 
sources (see, for examples, [6-10]), but no satisfactory conventional solutions has been found up till now.

The conservation laws associated with related to continuous symmetries date back to the pioneering work of G.Weyl $[11,12]$. The conservation laws associated with discrete symmetries are adjacent to the continuous symmetry, but they, to some extent, are different in their ideology.

Now let us turn to our point of view. We show that the usual three-dimensional space, in general, does not possess mirror symmetry with respect to the conversion, and this very fact explains the nonconservation of spatial parity.

Usually, when considering the mathematical formalism which is associated with the concept of parity it is mirror symmetry is mentioned in passing, and we go straight to the spatial inversion, which is equivalent to the reflection in three mutually perpendicular mirrors (see, for examples, $[13,14])$. Thus, in our opinion some essential points are lost. We will discuss the mirror reflection in one mirror in more detail. We will call it a single mirror transformation.

First of all, let us discuss the mirror transformation component of true (polar) and pseudovectors (axial vector) which a perpendicular and parallel to the surface of the mirror. We mark true vector as $\boldsymbol{A}$, pseudovector as $\boldsymbol{B}$, the point of application of the vectors $\boldsymbol{A}$ and $\boldsymbol{B}$ as $O_{A}$ and $O_{B}$. Strokes will be used to mark the same values after the mirror transformation. Let us consider the transformation of pseudovector which is perpendicular to the mirror surface (Fig. 1).

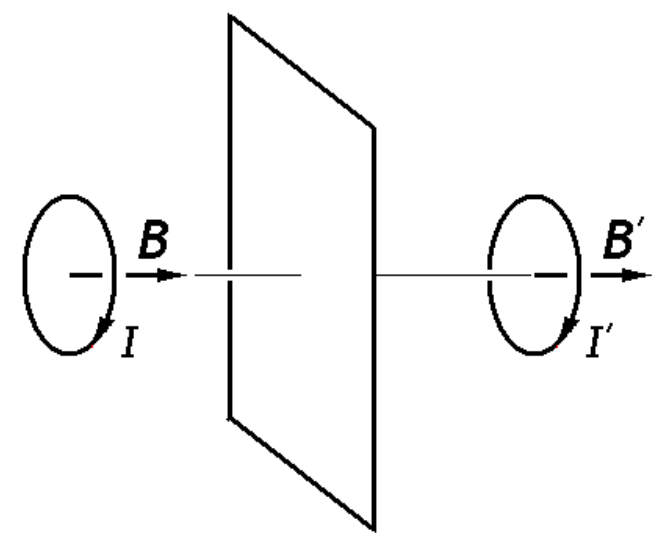

Fig. 1. Mirror transformation of the pseudovector which is perpendicular to the surface of the mirror

Let us suppose, for example, before the mirror (in Fig. 1 left) there is a circular coil with a current $I$, which is the source of the magnetic field $\boldsymbol{B}$ (Fig. 1, on the right shows the pattern behind the mirror). Thus, the pseudovector which is perpendicular to the surface of the mirror, in 
the mirror transformation does not change the direction. It is well known that the pseudovector which is parallel to the surface of the mirror changes its direction for the reversed in the mirror transformation. For the true vector the situation is reversed. Let us mark the component of the vector which is parallel to the surface of the mirror as index 1 and the perpendicular component is index 2. Thus, we have the following laws of the mirror transformation:

$$
A_{1}^{\prime}=A_{1} ; A_{2}^{\prime}=-A_{2} ; B_{1}^{\prime}=-B_{1} ; B_{2}^{\prime}=B_{2}
$$

As we can see, in terms of the mirror transformation both true vectors and pseudovectors are possess equal rights in case we swap their perpendicular and parallel components (Fig. 2).

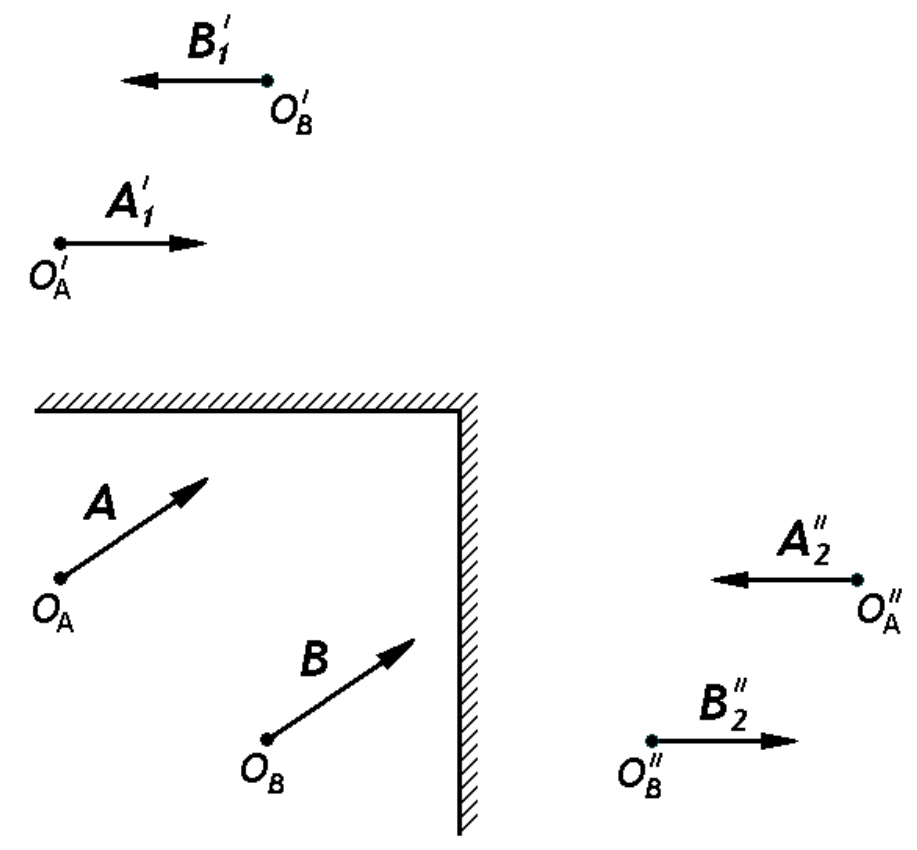

Fig. 2. Mirror transformation of the parallel and perpendicular components of the true vector and pseudovector

If we consider the transformation of the perpendicular and parallel components of the true vector and the pseudovector and then add them up, we get the following picture (Fig. 3). 

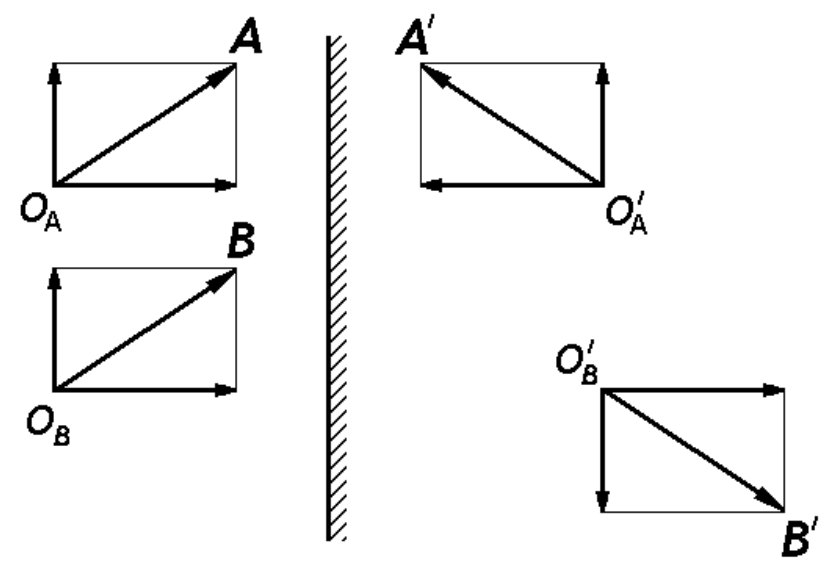

Fig. 3. Mirror transformation of the true vector and pseudovector

Thus, if the real experimental situation is depicted by a set of true vectors and pseudovectors than the mirror transformation of the whole picture is not symmetric.

Now let us consider the operation of space inversion: $x \rightarrow-x, \quad y \rightarrow-y, \quad z \rightarrow-z$. Otherwise it can be interpreted as mirror reflection in the three mutually perpendicular mirrors (the order of such reflections is not important, all the options lead to the same result). The transformation of true vectors and pseudovectors under spatial inversion is shown in Fig. 4.

Here is some explanations. The lower left part of the figure is our usual space, in which there is a true vector and pseudovector. The bottom right of the figure is the picture after the first mirror transformation. The upper right part of the figure is the picture after the second mirror transformation in the mirror, which is perpendicular to the first one.

In this part the dotted line depicts the third mirror, which is perpendicular to the first two mirrors. This mirror is parallel to the plane of the figure, but it lies under this plane. 

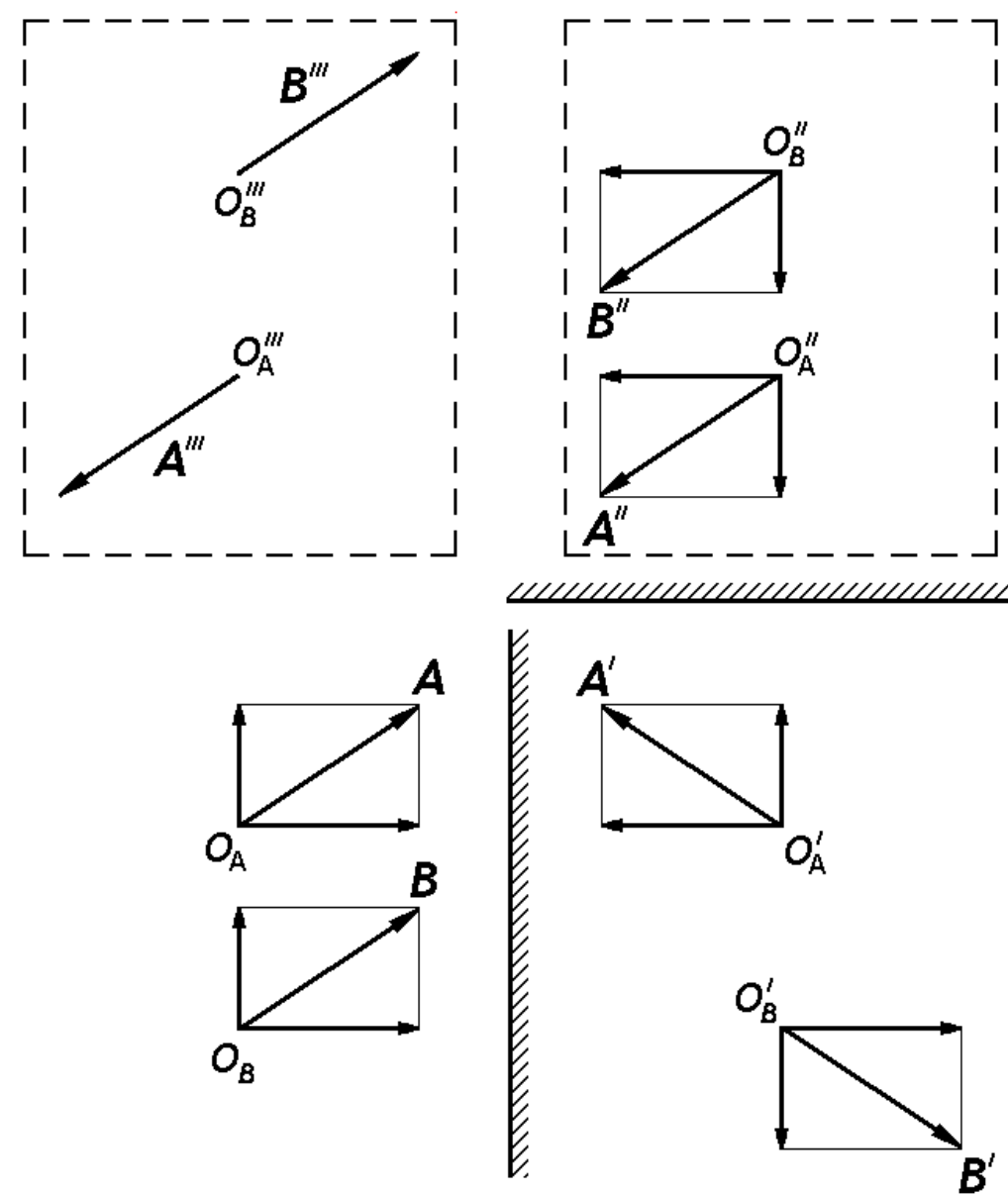

Fig. 4. Spatial inversion of the true vector and pseudovector

In the upper left part of the figure shows the picture after the third mirror transformation in dotted mirror. This picture is also parallel to the figure plane, but lies below it, i.e. under the third mirror.

As we see, if the real experimental situation described by a set of true vectors and pseudovectors, then the initial and the final picture are not symmetric with respect to each other.

Thus, both single mirror transformation and spatial inversion in the presence of a set of true vectors and pseudovectors does not possess any symmetry.

Thus, the question "Why $P$-parity is conserved in a particular process?" is more assential than the question "Why $P$-parity is not conserved?". From this point of view the search for the nonconservation of $P$-parity in the process with strong and electromagnetic interactions makes sense. By the way, if the weak and electromagnetic interactions have already been combined into the electroweak, then why does not the nonconservation of $P$-parity in the electromagnetic interaction take place, if it exists in the weak one? 
Based on the abovementioned considerations, let us consider the fundamental experiment of C.Vu [2] (Fig. 5).
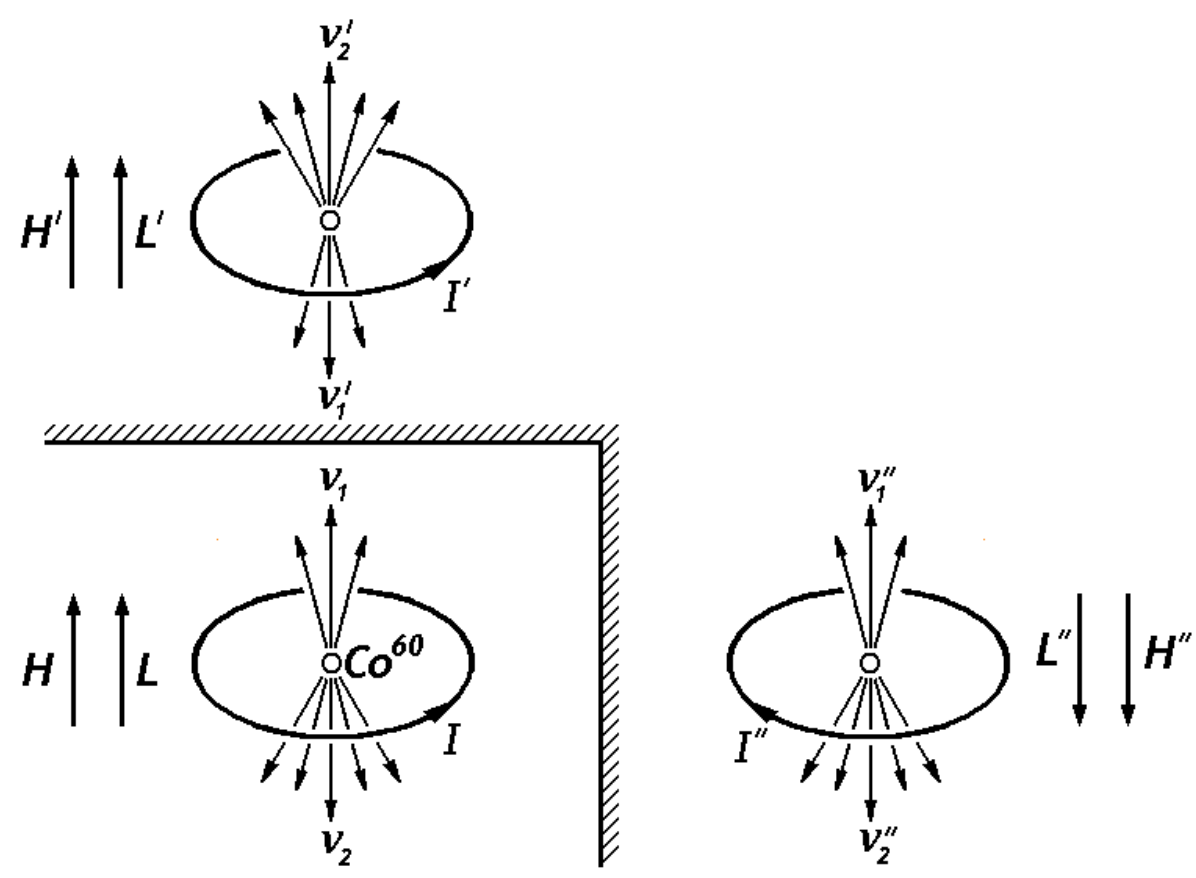

Fig.5. The experiment of C.Wu (1957) on the detection of nonconservation of P-parity in the weak interactions at $\beta$-decay of cobalt

This experiment investigated the angular distribution of electrons emitted by the radioactive decay of cobalt.

$$
{ }_{27} \mathrm{Co}^{60} \rightarrow{ }_{28} \mathrm{Ni}^{60}+\bar{e}+\tilde{v}
$$

A sample of cobalt was placed in a strong magnetic field, which oriented the spins of the nucleus of cobalt. The process was investigated at temperature close to zero, so that the thermal collision does not violate the orientation of the spins. The experiment discovers that in the direction opposite to the spin of the nucleus the number of emitted electrons is approximately $40 \%$ more than the number of electrons emitted in the same direction with the spin.

How is this experiment interpreted usually? It was stated, that if the same number of electrons emitted in the same and opposite directions of the spin of nucleus the mirror symmetry 
would be observed and the law of conservation of $P$-parity would be fulfilled. But the experiment showed a different numbers of electrons.

We dare to make the statement that even if the same numbers of electrons no mirror symmetry is observed. Let us consider this fact in more detail. In the initial picture (left lower part of Fig. 5) the velocity vector of electrons $\boldsymbol{v}_{2}$ is antiparallel to the magnetic field vector and to the spin of cobalt nucleus, and the vector $\boldsymbol{v}_{1}$ - parallel to them.

In the mirror transformed picture (upper left and bottom right of Fig. 5), the situation is reversed: the direction of the vector $\boldsymbol{v}_{2}$ is parallel to the vector of the magnetic field and the spin of the nucleus, and the vector $\boldsymbol{v}_{1}$ is antiparallel to them.

The experiment show that the electrons emitted with the at speeds $\boldsymbol{v}_{1}$ and $\boldsymbol{v}_{2}$, are recorded on a macroscopic distance from each other. Therefore, the probability density of detecting them are practically not overlapped, i.e. the electrons are in different systems, and because the quantummechanical principle of identity and indistinguishability does not work for them.

Thus, the electrons are emitted with speeds $\boldsymbol{v}_{1}$ and $\boldsymbol{v}_{2}$, are quite different and distinguishable even if all their parameters (speed module, the beam intensity and the angular distribution) are equal. So, even if the same parameters of electron beams emitted upwards and downwards (in Figure 5) in the mirror, strange as it may seem at first glance, is not a mirror symmetry. It is more true for the beams with different parameters. So we state that both pseudovectors and true vectors can depict real objects.

So, here is the main conclusion of the paper. Both theoretical analysis and experiments show, that if the real situation is described by a combination of the true vectors and pseudovectors, the reflection in the mirror is not mirror symmetry.

In conclusion we consider the transformation of the electromagnetic field tensor in single mirror reflection and in spatial inversion. As it is known, the antisymmetric tensor of the electromagnetic field is bivector which consists of the true electric field vector and pseudovector of the magnetic field.

In the following formula the first arrow denotes mirror transformation in the plane perpendicular to the axis $x$, the second arrow in the plane which perpendicular to the axis $y$, and the third - the axis $z$. 


$$
\begin{gathered}
\left\{\begin{array}{cccc}
0 & c B_{z} & -c B_{y} & -i E_{x} \\
-c B_{z} & 0 & c B_{x} & -i E_{y} \\
c B_{y} & -c B_{x} & 0 & -i E_{z} \\
i E_{x} & i E_{y} & i E_{z} & 0
\end{array}\right\} \rightarrow\left\{\begin{array}{cccc}
0 & -c B_{z} & c B_{y} & i E_{x} \\
c B_{z} & 0 & c B_{x} & -i E_{y} \\
-c B_{y} & -c B_{x} & 0 & -i E_{z} \\
-i E_{x} & i E_{y} & i E_{z} & 0
\end{array}\right\} \rightarrow \\
\rightarrow\left\{\begin{array}{cccccc}
0 & c B_{z} & c B_{y} & i E_{x} \\
-c B_{z} & 0 & -c B_{x} & i E_{y} \\
-c B_{y} & c B_{x} & 0 & -i E_{z} \\
-i E_{x}-i E_{y} & i E_{z} & 0
\end{array}\right\} \rightarrow\left\{\begin{array}{cccc}
0 & c B_{z} & -c B_{y} & i E_{x} \\
-c B_{z} & 0 & c B_{x} & i E_{y} \\
c B_{y}-c B_{x} & 0 & i E_{z} \\
-i E_{x}-i E_{y} & -i E_{z} & 0
\end{array}\right\}
\end{gathered}
$$

Thus, both with a single mirror transformation and in the spatial inversion the initial and final pictures are not symmetric with respect to each other.

Each mirror transformation changes the sign in half of the components (in three out of six). I the spatial inversion the components of the magnetic field change their sign twice (as a result they remain with the same sign) and the components of the electric field change their sign once.

\section{References}

1. Lee T.D., Yang C.N. (1956). Proposals to test spatial parity conservation in weak interactions. The Physical Review, Vol. 104, No. 1, 254-258.

2. Wu C.S., Ambler E., Hayward R.W. (1957). Experimental test of parity conservation in beta decay. The Physical Review, Vol. 105, 1413-1415.

3.Wigner E.P. (1971). Symmetries and Reflections. Moscow: MIR [World].

4. Lee T.D., Yang C.N. (1957). In the collection: New symmetry properties of elementary particles. Moscow: World, 13-25.

5. Christensen J.H., Cronin J.W. (1964). Evidence for the 2 pi decay of the K20 Meson. Phys.Rev.Lett., Vol. 13, 138-140.

6. Gibson W.M, Pollard B.R. (1979). Symmetry Principles in Elementary Particle Physics. Moscow: Atompress.

7. Okun L.B. (1990). Leptons and Quarks. Moscow: Nauka [Science].

8. Kane G. (1990). Modern Elementary Particle Physics. Moscow: World.

9. Chelnokov M.B. (2010). On Spin of Fundamental Particles. Vestnik MGTU. Estestvennye nauki [Herald of BMSTU. Natural science], № 3 (38), 22-34. 
Proceedings of International Conference PIRT-2015

10. Chelnokov M.B. (2010). On Spin Projection of Fundamental Particles and Problem of NonConservation of CP-Parity. Vestnik MGTU. Estestvennye nauki [Herald of BMSTU. Natural science], № 4 (39), 73-85.

11. Weyl G. (1947). Classic groups, invariants and representations. Moscow: Foreign Literature. 12. Weyl G. (1968). Symmetry. Moscow: Nauka [Science].

13. Bogolyubov N.N., Shirkov D.V. (1980). Quantum Fields. Moscow, Nauka [Science].

14. Bogolyubov N.N., Shirkov D.V. (1984). Introduction in Theory of Quantum Fields. Moscow: Nauka [Science]. 